\title{
Sobre a relação entre a energia da luz e sua frequência
}

On the relation between the energy of light and its frequency

\author{
Jayme F.M. Mendes* \\ Instituto Militar de Engenharia (IME) \\ Praça Gen. Tibúrcio, 80, Urca, \\ Rio de Janeiro - RJ - 22290-270
}

\begin{abstract}
Consideramos aqui a absorção de ondas eletromagnéticas monocromáticas por um corpo massivo em repouso. O processo é analisado em dois referenciais inerciais quanto à conservação de energia relativística e efeito Doppler relativístico transversal. A comparação entre a energia da onda eletromagnética e sua respectiva frequência nos dois referenciais resulta em uma invariância relativística para a razão 'energia da onda eletromagnética'/'frequência'.
\end{abstract}

Palavras-chaves: Onda Eletromagnética; Invariância Relativística; Energia Eletromagnética; Frequência.

We consider here the total absorption of monochromatic electromagnetic waves by a massive body at rest. The process is analyzed in two inertial reference frames with respect to relativistic Doppler effect and energy conservation. The comparison between the energy of the electromagnetic wave and their respective frequency in each reference system results in a relativistic invariance for the ratio 'electromagnetic wave energy'/'frequency'.

Key-words: Electromagnetic Wave; Relativistic Invariance; Electromagnetic Energy; Frequency.

\section{INTRODUCTION}

Desde a proposta de Einstein para explicar o efeito fotoelétrico [1] a partir dos trabalhos de Max Planck para a radiação de corpo negro [2], até o desenvolvimento da eletrodinâmica quântica, as tentativas de obtenção da expressão para a energia de uma onda eletromagnética como igual ao produto de uma constante pela sua frequência não foram bem sucedidas.

Neste artigo simples, realizamos o cálculo, em dois referenciais de Lorentz, para a energia de uma onda eletromagnética monocromática a partir da sua absorção por um corpo massivo em repouso.

A intenção é obter a razão entre a energia da onda e sua frequência nos dois referenciais e verificar se o resultado é um invariante relativístico ou não. Mais ainda, caso seja um

*Endereço Eletrônico: jayme.felipe@gmail.com invariante devemos verificar, também, se este resultado é, ou não, dependente de grandezas físicas.

A Figura [1 mostra dois feixes de luz de mesma frequência $f$ e mesma energia $E$ no referencial $S$, antes de serem absorvidos simultaneamente pelo corpo em repouso de massa $m_{\circ 1}$. A luz considerada é linearmente polarizada em uma direção ortogonal à da velocidade relativa entre os referenciais $S$ e $S^{\prime}$.

Após a absorção, o corpo permanece em repouso em $S$, pois o momento total do sistema é nulo. Sendo assim, a energia total do sistema após a absorção da luz é dada apenas em função da nova massa de repouso: $m_{\circ 2} c^{2}$. A fim de comparar os valores da energia da radiação sob o ponto de vista de observadores em dois referenciais inerciais, $S$ e $S^{\prime}$, analisemos a absorção nesses dois casos.

A conservação da energia em $S$ se expressa:

$$
E_{i}=E_{f},
$$


onde,

$$
E_{i}=m_{\circ 1} c^{2}+2 E \quad E_{f}=m_{\circ 2} c^{2} .
$$

A conservação da energia em $S^{\prime}$, por sua vez, fornece:

$$
E_{i}^{\prime}=E_{f}^{\prime}
$$

com,

$$
E_{i}^{\prime}=\gamma m_{\circ 1} c^{2}+2 E^{\prime} \quad E_{f}^{\prime}=m^{\prime} c^{2}
$$

Uma vez que o corpo permanece em repouso

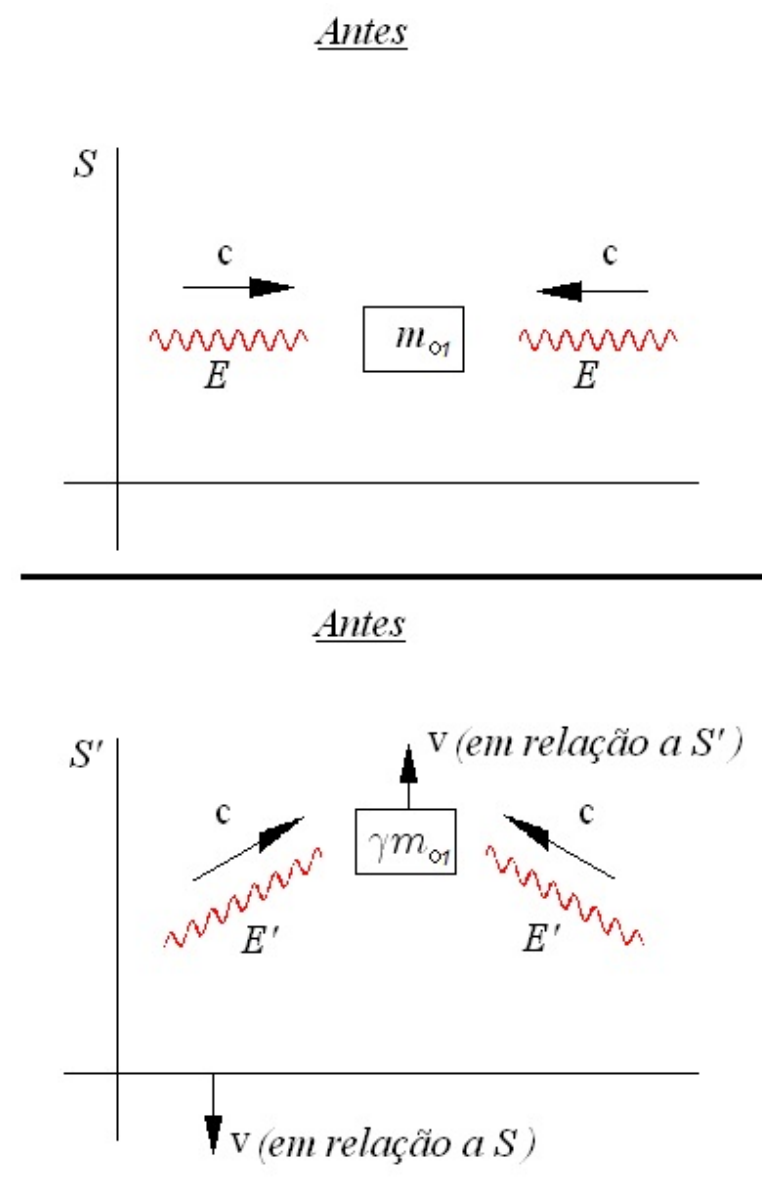

Definindo as razões entre as energias das on- após a absorção em $S$, para um observador no referencial $S^{\prime}$ a sua velocidade também não poderá ser alterada após a absorção, portanto, mantém-se igual a $v$.

Do que foi dito acima resulta:

$$
m^{\prime}=\gamma m_{\circ 2}
$$

com

$$
\gamma=\frac{1}{\sqrt{1-\frac{v^{2}}{c^{2}}}}
$$

$\underline{\text { Depois }}$
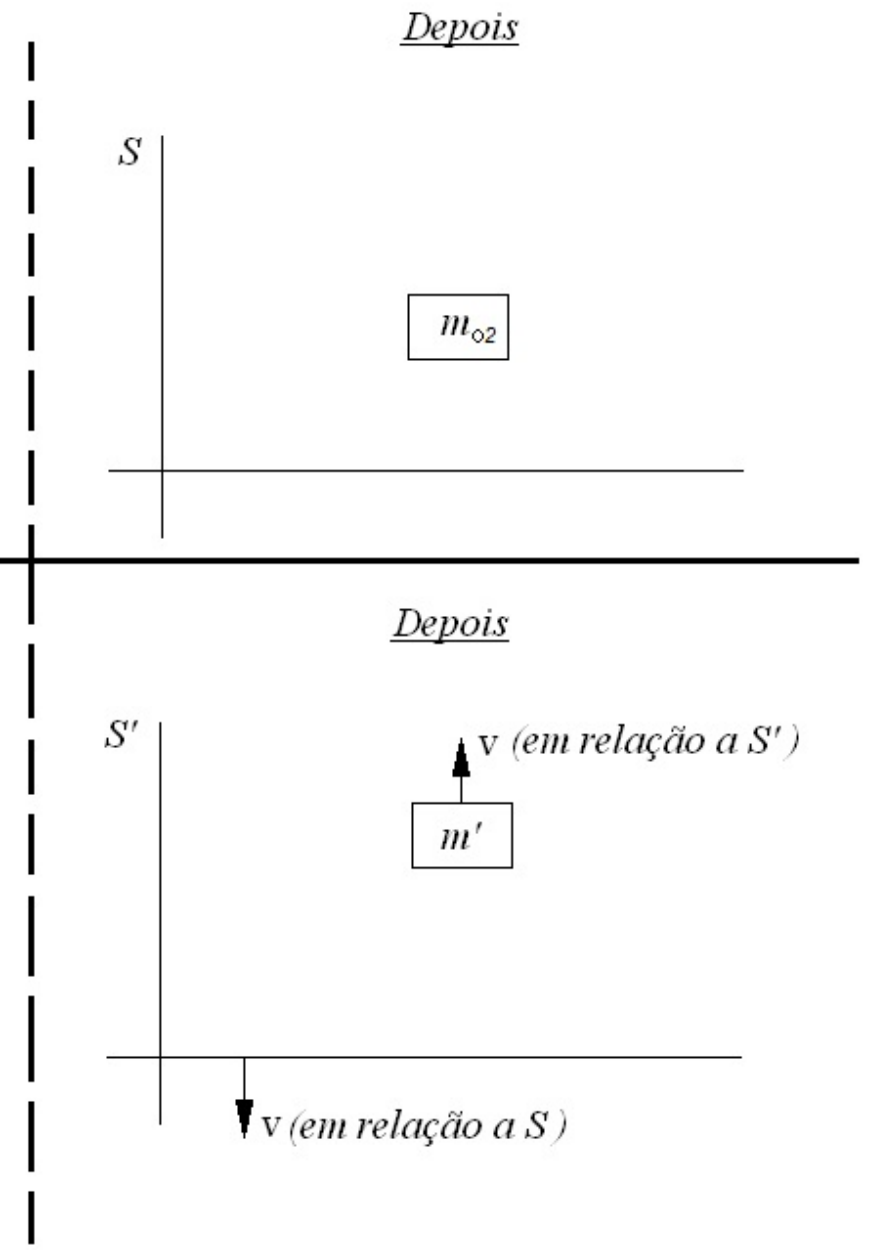

$$
g=\frac{E}{f}, \quad g^{\prime}=\frac{E^{\prime}}{f^{\prime}}
$$


pode-se reescrever as equações de conservação:

- no referencial $S$ :

$$
m_{\circ 1} c^{2}+2 g f=m_{\circ 2} c^{2},
$$

- enquanto que, no referencial $S^{\prime}$ :

$$
\gamma m_{\circ 1} c^{2}+2 g^{\prime} f^{\prime}=\gamma m_{\circ 2} c^{2} .
$$

Das equações (6) e (7) resulta,

$$
\gamma m_{\circ 1} c^{2}+2 g^{\prime} f^{\prime}=\gamma\left(m_{\circ 1} c^{2}+2 g f\right) .
$$

ou ainda, simplificando,

$$
g^{\prime} f^{\prime}=\gamma g f
$$

Estas mesmas frequências surgem em uma outra equação quando se considera o efeito Doppler relativístico transversal, exatamente o observado por um observador em $S^{\prime}$ em relação à onda com frequência $f$ em $S$. Neste caso, o efeito mostra que [5],

$$
f^{\prime}=\gamma f
$$

Portanto, segue das equações (9) e (10),

$$
g^{\prime} \gamma f=\gamma g f
$$

ou seja,

$$
g^{\prime}=g,
$$

onde $g^{\prime}$ está associado a um referencial $S^{\prime}$, cuja velocidade $v$ em relação ao referencial $S$ é arbitrária. Se considerássemos um terceiro referencial com velocidade $v^{\prime}$ em relação a $S$ chegaríamos a $g^{\prime \prime}=g$ e, consequentemente, $g=g^{\prime}=g^{\prime \prime}$. Sendo assim, $g$ é um invariante relativístico.

O resultado, portanto, é que a energia da onda em um dado referencial pode ser dada simplesmente pelo produto de um invariante pela sua respectiva frequência:

$$
E=g f \quad \text { e } \quad E^{\prime}=g f^{\prime} .
$$

Deve-se observar que a massa de repouso pode ser tomada tão pequena quanto se queira, mostrando assim que o corpo massivo não tem função essencial no processo físico em questão.

\section{ALGUMAS CONSIDERAÇÕES}

Uma pergunta deve ser feita sobre a equação $E=g f:$ sendo $g$ um invariante, a energia de uma onda eletromagnética pode ser dada apenas em função da sua frequência $f$ na forma $E=g f$ ? Ou seja, $g$ é uma constante no sentido de ser independente das grandezas que caracterizam a onda?

Para responder às perguntas acima, devemos considerar que as grandezas físicas que caracterizam completamente uma onda eletromagnética plana são a frequência, a amplitude do campo elétrico e o estado de polarização. Considerando que a frequência não é um invariante, assim como o campo elétrico em questão, pois a onda está linearmente polarizada em uma direção perpendicular à direção de deslocamento entre os referenciais, o invariante $g$ não pode depender de nenhuma grandeza física da onda e, portanto, é um invariante dado por uma constante.

Naturalmente, tomando um feixe de secção reta finita $A$ da onda ao longo de um comprimento $\Delta \ell=c \Delta t$, a constante $g$ deverá tender a zero quando $\Delta t \rightarrow 0$ e/ou $A \rightarrow 0$. Esta última consideração, além da expressão $E=g f$, também é consistente com a interpretação corpuscular da luz onde, em um feixe monocromático uniforme teríamos em um volume $A c \Delta t$ a energia sendo igual ao produto de um invariante pela frequência $f$ na forma:

$$
E=n h f, \quad n=0,1,2,3 \ldots
$$

Eis a relação! 
(6) 132 (1905).

[2] M. Planck, Zur Theorie des Gesetzes der Energieverteilung im Normalspektrum. Verhandlungen der Deutschen Physikalischen Gesselschaft 2, 237 (1900).

[3] A. Einstein, Ist die Trägheit eines Körpers von seinem Energieinhalt abhängig? Ann. Phys. (Berlin) 18, (13) 639 (1905).
[4] A. Einstein, Zur Elektrodynamik bewegter Körper. Ann. Phys. (Berlin) 17, (10) 891 (1905).

[5] J.D. Jackson, Classical Eletrodynamics (3rd Edition). New Jersey: John Wiley \& Sons (1998). 